ISSN 2011 - 835X (printed)

ISSN 2463 - 1965 (online)
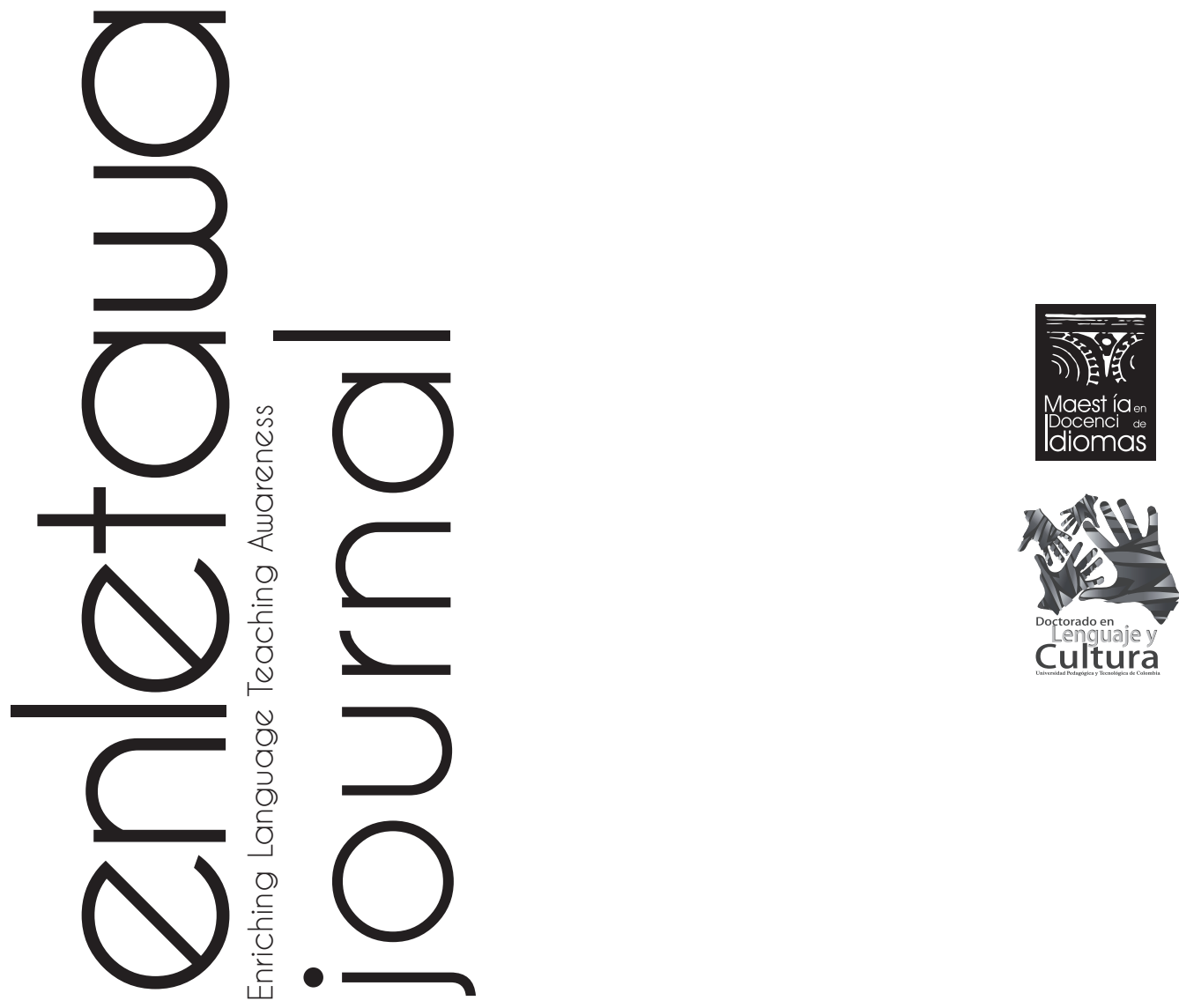

UNIVERSIDAD PEDAGÓGICA Y TECNOLÓGICA DE COLOMBIA MAESTRÍA EN DOCENCIA DE IDIOMAS

Vol. 10, No. 1, JANUARY - JUNE - 2017

TUNJA - BOYACÁ - COLOMBIA 


\title{
enletawa journal
}

\author{
Enriching Language Teaching Awareness \\ ISSN 2011-835X (printed) ISSN 2463- 1965 (online)
}

Enletawa Journal is an academic publication of the Masters in Language Teaching at Universidad Pedagógica y Tecnológica de Colombia. The Journal provides a means of dissemination of academic research, pedagogical innovation, and theoretical discussion articles. Enletawa Journal is a biannual publication that receives articles in English, French and/or Spanish. For comments, contributions or journal exchanges please write to Maestría en Docencia de Idiomas, UPTC Avenida Central del Norte 39 - 115 Edificio Central, office C-224; PBX: (+57) 87405626 Ext. 2470. http://revista.uptc.edu.co/revista/revistas/index.php/enletawa_journal1\%20

\section{Legal Status}

Copyright

Enletawa Journal

(C) Master's in Language Teaching / Faculty of Education Sciences. Universidad Pedagógica y Tecnológica de Colombia.

This publication rights of articles in each edition belong to the Master's in Language Teaching at Universidad Pedagógica y Tecnológica de Colombia. Copy and material quotation of the journal are authorized when it is mentioned specifically; journal's name, author's (s) name, year, number and pages of the article. The mention of commercial products or firms in the journal does not imply a recommendation or support by the university or the Master's program, the use of those products must adhere to label recommendations.

Frequency: Biannual

Editor: Yuranny Marcela Romero Archila yuranny.romero@uptc.edu.co

\section{Design and Layout}

Andrés A. López Ramírez

anlopezram@gmail.com

Printing: PUBLIVISIÓN Artes Gráficas

Calle 6 No. 12-51, interior 2

Cel: 3103331019

publivisionartesgraficas@hotmail.com

Tunja - Boyacá - Colombia

\section{Acquisition, exchange and subscription:}

Universidad Pedagógica y Tecnológica de Colombia, Faculty of Education Sciences, Masters in Language Teaching. Avenida Central Norte 39-115, oficina C224.

revista.enletawajournal@uptc.edu.co

Telephone: (57) (8) 7405626. Ext. 2470

This journal can be found in all the libraries at Universidad Pedagógica y Tecnológica de Colombia and in both, national and international institutions in agreement with the University; It can also be found at websites:

http:/ / www.uptc.edu.co

https://www.uptc.edu.co/enlaces/reletawa

http://revistas.uptc.edu.co/revistas/index.php/ enletawa_journal

\section{Cost copy:}

Teachers: \$ 18.000.oo; Students: \$13.000.oo

\section{Responsibility}

1. The opinions expressed and the contents of the articles are the exclusive responsibility of their authors and do not necessarily reflect the view of the Editor, the Master's program, Enletawa Journal or UPTC.

2. The submission of an article to this journal does not imply a commitment to publish it. Any article published in the Journal may be quoted as long as the source is clearly referenced.

Enletawa Journal is indexed in:

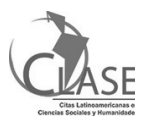




\section{EDITOR}

M.A. Yuranny Marcela Romero Archila

Universidad Pedagógica y Tecnológica de Colombia, Colombia

\section{EDITORIAL COMMITTEE}

Ph.D. José Aldemar Álvarez Valencia Universidad del Valle, Colombia

\section{M.A. Bertha Ramos Holguín}

Universidad Pedagógica y Tecnológica de Colombia, Colombia

\section{PROOFREADER}

\section{Anna Carolina Peñaloza}

\section{ADMINISTRATIVE SERVICES ASSISTANT}

Isabel Esquivel de Parra

Universidad Pedagógica y Tecnológica de Colombia, Colombia

\section{SCIENTIFIC COMMITTEE}

\section{M.A. Trudie Clarck McEvoy}

University of Arizona, USA

Ph.D. A.G. Rud

Washington State University, USA

Ph.D. Alberto Fajardo Castañeda

Universidad Pedagógica y Tecnológica de Colombia, Colombia

M.A. Álvaro Hernán Quintero Polo Universidad Distrital Francisco José de Caldas, Colombia

M.A. Carlo Erwin Granados Beltrán Institución Universitaria Colombo Americana (ÚNICA), Colombia

\section{EDITORIAL REVIEW BOARD}

We would like to thank the following professors for their valuable time reviewing the articles:

\section{M.A. Alberto Ramírez Avendaño}

Universidad Pedagógica y Tecnológica de Colombia, Colombia

M.A. Álvaro Hernán Quintero Polo

Universidad Distrital Francisco José de Caldas, Colombia

B.A. Anna Carson

Washington and Lee University, United States

M.A. Bertha Ramos Holguín

Universidad Pedagógica y Tecnológica de Colombia, Colombia
M.A. Carlo Erwin Granados Beltrán

Institución Universitaria Colombo Americana

(ÚNICA), Colombia

M.A. Carol Anne Ochoa Alpala

Universidad Santo Tomás, Colombia

M.A. Jahir Aguirre Morales

Universidad Pedagógica y Tecnológica de Colombia, Colombia

Ph.D. Ken Beatty

Anaheim University, United States

M.A. Lucimaver González Robayo

Universidad Santo Tomás, Colombia

M.A. María Teresa Esteban Núñez

Universidad Pedagógica y Tecnológica de Colombia, Colombia

\section{DIRECTIVES}

\section{RECTOR}

Dr. Alfonso López Díaz

Universidad Pedagógica y Tecnológica de Colombia, Colombia

\section{ACADEMIC VICE-RECTOR}

Dr. Hugo Alfonso Rojas Sarmiento

Universidad Pedagógica y Tecnológica de Colombia, Colombia

\section{RESEARCH DIRECTOR - DIN}

Dr. Enrique Vera López

Universidad Pedagógica y Tecnológica de Colombia, Colombia

\section{HEAD OF THE SCHOOL OF LANGUAGES}

M.A. Sonia Marisol Rojas Espitia

Universidad Pedagógica y Tecnológica de Colombia, Colombia

\section{DEAN OF THE FACULTY OF EDUCATION}

Dra. Diana Elvira Soto Arango

Universidad Pedagógica y Tecnológica de Colombia, Colombia

\section{DIRECTOR OF THE MASTER'S IN LANGUAGE TEACHING}

M.A. Yuranny Marcela Romero Archila

Universidad Pedagógica y Tecnológica de Colombia, Colombia 

Enletawa Journal n. 10.1

ISSN 2011 - 835X (printed)

ISSN 2463 - 1965 (online)

January - June - 2017

\section{Contents}

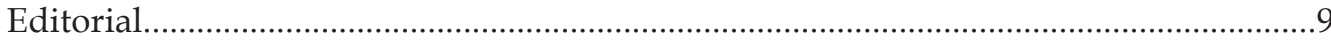

Yuranny Marcela Romero Archila

\section{Theme Review}

Globalization, Cultural Diversity, Education. .13 Enrique Vez López

\section{Research Reports}

Critical Analysis of Advertising: Enhancing Identity Construction in EFL

classrooms.

Mónica Yohanna Lara Páez

Feedback: understanding strategies and impact on EFL

learning and teaching contexts.

David Felipe Espinosa Torres

Iván Camilo González Bejarano

Juliana Moreno Restrepo

\section{Pedagogical Experiences}

Using Mobile Learning to Enhance Pre-Service Teachers' Participation in

Distance Education English Tutoring Sessions.

Angélica María Carvajal Téllez

Yolanda Duarte Medina

\section{Reflective Papers}

Reflections on student-centered learning: An alternative to traditional

Englishclasses.

Sulma Patricia Fonseca Cely

Leidy Girleza Cano García 

Enletawa Journal $n^{\circ} .10 .1$

ISSN 2011 - 835X (printed)

ISSN 2463 - 1965 (online)

January - June - 2017

\section{Contenidos}

Editorial 9

Yuranny Marcela Romero Archila

\section{Revisión de tema}

Globalización, Diversidad Cultural, Educación.

Enrique Vez López

\section{Reportes de Investigación}

Análisis Crítico de la Publicidad: Fortaleciendo la Construcción de Identidad en

Clases de Inglés como Lengua Extranjera.

Mónica Yohanna Lara Páez

Re-alimentación: Comprensión de estrategias y su impacto en contextos de aprendizaje y enseñanza del Inglés como lengua extranjera.

David Felipe Espinosa Torres

Iván Camilo González Bejarano

Juliana Moreno Restrepo

\section{Experiencias Pedagógicas}

Aprendizaje Móvil para mejorar la participación de los docentes en formación en sesiones tutoriales en Educación a Distancia.

Angélica María Carvajal Téllez

Yolanda Duarte Medina

\section{Artículos reflexivos}

Reflexiones sobre el enfoque centrado en el estudiante: Una alternativa

para las clases tradicionales de inglés

Sulma Patricia Fonseca Cely

Leidy Girleza Cano García 